\title{
National survey of methadone prescribing for maintenance treatment: 'opiophobia' among substance misuse services?
}

\section{AIMS AND METHOD}

The aims of this study were to describe the characteristics of substance misuse services prescribing methadone for maintenance treatment of opioid dependence and to determine the average daily doses of methadone prescribed across England. A postal questionnaire survey of all substance misuse treatment centres in England was carried out.

\section{RESULTS}

A total of 298 treatment centres were identified and contacted, 212 of which responded to the survey

\author{
(response rate of $71 \%$ ). Of these, 157 \\ were prescribing methadone for \\ maintenance treatment; the majority \\ (71\%) were community-based and 125 \\ centres had doctors attached. The \\ most common formulation of \\ methadone prescription was oral \\ methadone mixture (152 centres, \\ $97 \%$ ). The mean daily dose of \\ methadone prescribed was $47 \mathrm{mg}$. \\ Surprisingly, 37 (24\%) of the res- \\ pondents felt that methadone \\ maintenance treatment should be \\ time-limited and 21 teams (13\%) \\ stated that patients should receive \\ only drug substitution and no \\ psychosocial interventions.
}

\begin{abstract}
CLINICAL IMPLICATIONS
There is currently a move among substance misuse services towards community-based treatment. In our survey, the mean daily dose of methadone prescribed was less than the dosage recommended by the Department of Health. This suggests that specialist addiction services are continuing to underprescribe heroin substitutes. The inadequate understanding of some of the respondents of the basic principles of methadone maintenance treatment also raises concerns, and highlights the need for further training and education.
\end{abstract}

Methadone maintenance treatment is the most widely known, well-researched and cost-effective treatment for opioid dependence (Avants et al, 1999; Krambeer et al, 2001). There are several substance misuse services, which provide this treatment in differing settings across the UK. The settings, resources and the prescribing patterns vary among these services, although they all work towards the same goal of helping addicts refrain from using heroin. A coherent picture of these different services would be useful in understanding their pattern of functioning and would have important treatment implications.

Studies in the UK have shown that for patients on methadone maintenance, daily doses of over $50 \mathrm{mg}$ are associated with lower rates of heroin use (Department of Health, 1996). Research has also found that patients on higher doses of methadone have better outcomes (Farrell et al, 1994). Community pharmacy surveys of methadone prescribing in the UK reveal that over $50 \%$ of patients receive doses lower than $50 \mathrm{mg}$ (Strang \& Sheridan, $1998,2001)$. A recent survey of methadone prescribing at an inner-city drug service suggested that a significant minority of patients were on a sub-therapeutic dose (Dunn, 2003). These findings have led to concerns that opiate-dependent patients are being undertreated.

Despite several pharmacy surveys, nationwide studies based on gathering information from the treating team are few. An important limitation of such pharmacy surveys is that patients who receive two prescriptions for different formulations of methadone, such as ampoules and oral mixture, are counted twice. Also, 'patients on detoxification programmes, slow reduction regimes and methadone maintenance are grouped together in these studies, thus skewing the results to the left' (Dunn, 2003).

The aims of our study were to describe the characteristics of methadone maintenance treatment services across England, to determine the mean daily doses of methadone prescribed in maintenance treatments, and to determine the views of teams on the prescribing patterns.

\section{Method}

All treatment centres prescribing methadone for maintenance treatment of opiate-dependent patients were identified by searching the database of DrugScope (http://www.drugscope.org.uk). A questionnaire was developed by the researchers and a copy was sent by post, along with a covering letter to the team manager of each service in January 2004. Those who did not respond initially were sent a further copy 8 weeks later.

The questionnaire consisted of 18 questions, covering the following areas: the types of treatment services and medical staffing, methadone prescribing practices and the respondent's views on methadone prescribing. Simple descriptive analysis (mean and percentage calculations) was carried out on the numerical data obtained.

\section{Results}

Out of a total of 298 substance misuse treatment centres identified, 212 centres returned completed 


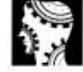

original papers
Table 1. Treatment services prescribing methadone for

maintenance in substance misuse

\begin{tabular}{lrr}
\hline Treatment service & $n(\%)$ \\
\hline Community drug teams & $112(71)$ \\
Addiction treatment units & $42(27)$ \\
General practices & 3 & $(2)$ \\
Total & $157(100)$ \\
\hline
\end{tabular}

questionnaires, giving a response rate of $71 \%$. Three centres had closed down. Of the remaining 209 centres, 157 (75\%) prescribed methadone for maintenance treatment of opiate addicts. Although the other centres prescribed methadone, this was for purposes other than for maintenance treatment, i.e. detoxification, slow reduction, etc.

\section{Types of treatment service and medical staffing}

The types of substance misuse treatment services that prescribed methadone for maintenance treatment are given in Table 1

A total of 125 centres (79.6\%) had doctors attached to the team. In 32 centres (20.4\%), the prescriptions were written by the local general practitioner (GP), who was not directly attached to the units. Forty-two (26.8\%) treatment centres had one doctor, 50 (31.8\%) had two doctors, 24 (15.3\%) had three doctors and 9 (5.7\%) had four doctors. In all, there were 68 consultant psychiatrists, 74 GPs, 78 staff and associate specialist doctors and 30 senior house officers. Altogether, 15931 patients were attending the 157 treatment centres for methadone maintenance treatment and on average, 101 patients were attending each centre (range $=2-1300$ ).

\section{Methadone prescribing practices}

The most common formulation of methadone prescribed was the oral methadone mixture, with 152 centres (97\%) prescribing this. Thirty-three centres $(21 \%)$ prescribed methadone tablets and 18 centres (11.5\%) prescribed injectable preparations in addition to the methadone mixture.

The maximum dose of methadone prescribed for maintenance ranged from 9 to $325 \mathrm{mg}$, with a mean of $116 \mathrm{mg}$. The minimum dose ranged from 1 to $70 \mathrm{mg}$. The mean daily dose for each centre was $47 \mathrm{mg}$ (range 9100). Seven centres (4\%) used serum levels to adjust the optimal dose, although these were used infrequently. None of the other 150 centres checked the serum levels of methadone.

\section{Respondents' views on methadone prescribing}

A total of $77(49 \%)$ centres expressed the view that there should be a maximum and a minimum dose of methadone that should be prescribed to an opiate-dependent patient; 63 (40\%) centres felt that methadone maintenance treatment should be restricted to specialised services; 37 (24\%) centres were of the opinion that methadone maintenance ought to be time-limited, but the majority disagreed with this; 21 centres (13\%) felt that opiate-dependent patients should receive only methadone, and no counselling or social interventions.

\section{Discussion}

There is currently a move among substance misuse services, in line with general psychiatric services, towards community-based treatment. This is evidenced by a high proportion of community teams found in this survey (71\%). The importance of non-statutory agencies in the treatment of opiate-dependent patients is highlighted by their significant numbers in our survey $(27 \%)$. The involvement of GPs was indicated by the large numbers involved in care (74 out of 250 doctors, 30\%).

In this survey, the proportion of services prescribing methadone tablets (21\%) and methadone ampoules $(11.5 \%)$ was higher than in other previous studies: the proportion of patients on methadone tablets in Dunn's (2003) survey was $17.2 \%$ and that in Strang \& Sheridan's (1998) survey was $9.5 \%$. This may be an indication that such preparations are available in many centres, but the actual number of patients prescribed them is low. Since drug formulation has no independent effect on illicit drug use, the choice could depend on availability and patient preferences. The Home Office (2000) document Reducing Drug Related Deaths, advises against the prescribing of controlled drugs to drug users because of the risk of injecting. It states that 'deaths would be reduced if agencies and GPs ceased to prescribe controlled drugs in tablet form or ampoules'.

We found that the mean daily dose of methadone per patient, per centre was $47 \mathrm{mg}$, which is lower than the Department of Health (1996) recommendation of a daily dose of over $50 \mathrm{mg}$. This finding is consistent with the findings from previous studies conducted in the UK (Strang \& Sheridan, 1998, 2001). International research shows that patients on higher doses of methadone show better outcomes (Ward et al, 1999). If lower doses mean poorer outcomes, the sub-optimal dose of methadone prescribing found in our survey is significant. Reasons remain unclear, although the fear of prescribing higher doses of methadone - 'opiophobia' - and lack of urgency in changing the prescription style may be important contributory factors.

Very few treatment centres (4\%) checked serum methadone levels as part of maintaining patients on methadone. It has been suggested that monitoring serum methadone levels is a much better way of determining the optimal dose of methadone than using arbitrary clinical judgement. Cost implications and resource limitations seem to be important practical considerations in this regard. Dunn (2003) argues that if its use were restricted only to patients who persistently use heroin 'on top' of 
their prescribed methadone, the cost would be less prohibitive.

The view expressed by nearly half ( $49 \%)$ the services that there should be a maximum and minimum dose limit for prescribed methadone is remarkable, as many centres continue prescribing sub-therapeutic doses. A substantial proportion $(24 \%)$ of centres in our survey felt that methadone maintenance should be time-limited. The opinion of $13 \%$ of services that patients should receive only methadone substitution and no psychosocial interventions requires further exploration. The benefits of offering methadone substitution as part of a psychosocial treatment package have been clearly demonstrated (Preston et al, 2000). Hence, our survey demonstrates that despite the commendable services offered by most teams, many require greater understanding of methadone maintenance treatment.

This national survey demonstrates that underprescribing of methadone for methadone maintenance treatment is still prevalent in many substance misuse services across England. This, combined with an inadequate understanding of the underpinning principles of methadone maintenance treatment by a substantial minority of treatment services, points to a need for further training of professionals involved in treating opioid-dependent patients.

\section{Acknowledgement}

We thank all the team managers who responded to this survey.

\section{Declaration of interest}

None.

original

papers

\section{References}

AVANTS, S. K., MARGIOLIN, A., SINDELAR, J. L., et al (1999) Day treatment versus enhanced standard methadone services for opioid dependant patients: a comparison of clinical efficacy and cost. American Journal of Psychiatry, 156, 27-33.

DEPARTMENT OF HEALTH (1996) The Task Force to Review Services for Drug Misusers. London: Stationery Office.

DUNN, J. (2003) A survey of methadone prescribing at an inner-city drug service and a comparison with national data. Psychiatric Bulletin, 27, 167-170.

FARRELL, M., WARE, J., MATTICK, R., et al (1994) Methadone maintenance treatment in opiate dependence: a review. BMJ, 309, 997-1001.

HOME OFFICE (2000) Reducing Drug Related Deaths, p. 72. London: Stationery Office.

KRAMBEER, L. L., VONMCKNELLY,W., Jr \& PENICK, E. C. (2001) Methadone therapy for opioid dependence.

Renju Joseph Specialist Registrar in General Adult Psychiatry, Lyndon Clinic, Hobs Meadow, Solihull, Birmingham B92 8RW, e-mail: renjujoseph@mail.com Hamdy F. Moselhy Consultant in Addiction Psychiatry, Sandwell Mental Health NHS \& Social CareTrust, West Bromwich 